\title{
SOME FEATURES OF DRAFTING OF CARGO PLAN OF CONTAINERSHIP
}

\section{НЕКОТОРЫЕ ОСОБЕННОСТИ СОСТАВЛЕНИЯ ГРУЗОВОГО ПЛАНА КОНТЕЙНЕРОВОЗОВ}

\author{
Ye. A. Vlasenko, senior lecturer, N. N. Tsymbal, DSc, professor \\ Е. А. Власенко, старший преподаватель, Н. Н. Цымбал, д.т.н., профессор \\ National University «Odessa Maritime Academy», Ukraine \\ Национальный университет «Одесская морская академия», Украина
}

\begin{abstract}
By the features of loading of containership which complicate the calculation of preliminary cargo plan, there is the necessity of account of rotary press of ports at the loading or unloading in a few ports and account of forces of inertia, affecting loading at tossing. If a ship adopts a loading which will unloading by parts in a few successive ports, on every transition between ports his nautical state will be different, that will lead to the change of forces of inertia, which will undergo a loading during transition.

At the loading of containership with the subsequent unloading in a few ports in the holds of ship it is necessary to produce placing of loading taking into account the sequence of ports of unloading, providing access to necessary parties of loading, and also taking to account that all transient states of loading must suit on stability, landing and durability of ship, and forces of inertia, arising up at tossing, must were in possible limits.

It is shown that forming of initial loading must be begun with determination of the possible last loading before handing over of loading in the last port, and then be incrementally moved to the previous loading, while the initial loading in the last port of loading will not be got.

At the decision the set problem it is necessary to take into account communication between the loading of ship and parameters of her tossing, which determine descriptions of nascent angular accelerations and influence on the size of forces of inertia.

To minimize the size of force of inertia operating on a container at tossing of ship, to the required legitimate values it is possible besides placing of container yet and by diminishment of peak value of angle of roll, for what it is necessary to obtain a maximal difference between frequency of own vibrations of ship and seeming frequency of agitation. It is shown that seeming frequency of agitation is an out of control variable and its size can be only forecast on forthcoming transition, however much the size of frequency of own vibrations of ship depends on the loading of ship. Exactly the loading of ship determines the size of transversal initial metacentric
\end{abstract}


height and moment of inertia of ship in relation to the longitudinal axis X-X. If from the great number of possible loadings to choose that, for which the difference of frequencies has the maximal value, other things being equal it is possible to provide implementation of requirement of legitimate values of forces of inertia at tossing of ship.

Keywords: nautical safety of ship, loading of ship, force of inertia on tossing.

\section{РЕФЕРАТ}

Особливостями завантаження контейнеровозів, які ускладнюють розрахунок попереднього вантажного плану, є необхідність урахування ротації портів при завантаженні або розвантаженні в декількох портах та сил інерції, які впливають на вантаж при качанні. Якщо судно приймає вантаж, який здається частинами в декількох послідовних портах, то на кожному переході між портами його морехідний стан буде різним, що поведе до зміни сил інерції, яким піддаватиметься вантаж під час переходу.

При завантаженні контейнеровоза 3 подальшим розвантаженням в декількох портах розміщення вантажу в трюмах судна слід проводити 3 урахуванням послідовності портів вивантаження, забезпечуючи доступ до необхідних партій вантажу, а також з урахуванням того, що всі проміжні стани завантаження повинні задовольняти вимогам по остійності, посадці і міцності судна, а сили інерції, виникаючі при качанні, повинні знаходилися в допустимих межах.

Показано, що формування початкового завантаження необхідно починати 3 визначення допустимого останнього завантаження перед здачею вантажу в останньому порту, а потім покроково переміщатися до попереднього завантаження, поки не буде одержане початкове завантаження в останньому порту завантаження.

При рішенні поставленої задачі слід враховувати зв'язок між завантаженням судна i параметрами його качання, які визначають характеристики виникаючих кутових прискорень і впливають на величину сил інерції.

Мінімізувати величину сили інерції, діючої на контейнер при качанні судна, до необхідних допустимих значень можна крім розміщення контейнера ще i зменшенням амплітудного значення кута крену, для чого необхідно добитися максимальної різниці між частотою власних коливань судна і уявною частотою хвилювання моря. Показано, що уявна частота хвилювання $\epsilon$ некерованою змінною і їі величину можна тільки прогнозувати на майбутній перехід, проте величина частоти власних коливань судна залежить від завантаження судна. Саме завантаження судна визначає величину поперечної початкової метацентричної висоти і моменту інерції судна щодо подовжньої осі $\mathrm{X}-\mathrm{X}$. Якщо з множини допустимих завантажень вибрати ту, для якої різниця частот має максимальне значення, то за інших рівних умов можна забезпечити виконання вимоги допустимих значень сил інерції при качанні судна. 
Ключові слова: морехідна безпека судна, завантаження судна, сили інерції на качанні.

\section{Постановка проблемы в общем виде и ее связь с важными научными или практическими задачами}

Особенностями загрузки контейнеровозов, которые усложняют расчет предварительного грузового плана, является необходимость учета ротации портов при загрузке или разгрузке в нескольких портах и учет сил инерции, воздействующих на груз при качке. Если судно принимает груз, который будет выгружаться частями в нескольких последовательных портах, то на каждом переходе между портами его мореходное состояние будет разным, что поведет к изменению сил инерции, которым будет подвергаться груз во время перехода. Во время каждого перехода параметры остойчивости, посадки и прочности судно должны находиться в допустимых пределах, а возникающие силы инерции от его качки, не должны превосходить допустимых пределов.

Анализ последних достижений и публикаций, в которых начато решение данной проблемы и выделение нерешенных ранее частей общей проблемы

Метод формирования оптимальной загрузки судна тарно-штучными грузами предложен в работе [1], а в работе [2] рассмотрен способ формирования оптимальной загрузки навалочных судов сыпучими грузами.

В работе [3] рассмотрена разработка метода оперативной оценки критериев мореходности судна с помощью имитационного моделирования. Процедура расчета угловых ускорений, возникающие при качке судна, изложена в статье $[4]$.

\section{Формулировка целей статьи}

Цель данной статьи - анализ некоторых особенностей составления предварительного грузового плана контейнеровоза, вызванных спецификой его работы.

Изложение основного материала исследования с обоснованием полученных научных результатов

При загрузке контейнеровоза с последующей разгрузкой в нескольких портах размещение груза в трюмах судна следует производить с учетом последовательности портов выгрузки, обеспечивая доступ к необходимым партиям груза, а также с учетом того, что все промежуточные состояния загрузки должны удовлетворять требованиям по остойчивости, посадке и прочности судна, а силы инерции, возникающие при качке, должны находились в допустимых пределах.

Более подробное рассмотрение этого вопроса позволяет предложить следующую формализацию загрузки судна. В первом приближении под загрузкой судна $\mathrm{G}$ будем понимать множество партий груза $\mathrm{p}_{\mathrm{i}}$, находящихся на судне, судовые запасы $\mathrm{P}_{\mathrm{z}}$, балласт $\mathrm{P}_{\mathrm{b}}$ и их размещение на судне $\mathrm{Q}$. Через $\widetilde{\mathrm{p}}_{\mathrm{i}}$ 
обозначим партию груза, к которой имеется свободный доступ для выгрузки, Поэтому формальная запись загрузки судна $\mathrm{G}$, как совокупности нагрузок от грузовых партий, балласта, судовых запасов и их размещения на судне:

$$
\mathrm{G}=\left\{\bigcup \mathrm{p}_{\mathrm{i}}, \mathrm{P}_{\mathrm{b}}, \mathrm{P}_{\mathrm{z}}, \mathrm{Q}\right\} .
$$

Начальная загрузка судна в порту отправления $\mathrm{G}_{\mathrm{o}}=\left\{\tilde{\mathrm{p}}_{\mathrm{l}} \cup \mathrm{p}_{\mathrm{i}}, \mathrm{P}_{\mathrm{b}}, \mathrm{P}_{\mathrm{z}}, \mathrm{Q}_{\mathrm{o}}\right\}$

должна обеспечить доступность к грузам, выгружаемым в первом порту разгрузки, обеспечить допустимое мореходное состояние $\mathrm{M}_{\mathrm{o}} \in \mathrm{Mn}_{\mathrm{sf}}$, где $\mathrm{Mn}_{\mathrm{sf}}$ - множество безопасных мореходных состояний, а значения сил инерции качки $\mathrm{F}_{\mathrm{jk}}$, действующих на груз, должны находиться в допустимых пределах, т. е. $\mathrm{F}_{\mathrm{jko}}<\mathrm{F}_{\mathrm{jd}}$. Целесообразно различать размещение груза $\mathrm{Q}_{\mathrm{c}}$ и балласта $\mathrm{Q}_{\mathrm{b}}$. Поэтому начальная загрузка судна:

$$
\begin{gathered}
\mathrm{G}_{\mathrm{o}}=\left\{\tilde{\mathrm{p}}_{1} \cup \mathrm{p}_{\mathrm{i}}, \mathrm{Q}_{\mathrm{co}}, \mathrm{P}_{\mathrm{z}}, \mathrm{P}_{\mathrm{bo}}, \mathrm{Q}_{\mathrm{bo}}\right\} \text { или } \\
\mathrm{G}_{\mathrm{o}}=\left\{\mathrm{G}_{\mathrm{co}}, \mathrm{P}_{\mathrm{bo}}, \mathrm{Q}_{\mathrm{bo}}\right\},
\end{gathered}
$$

где $\mathrm{G}_{\mathrm{co}}=\left\{\tilde{\mathrm{p}}_{1} \cup \mathrm{p}_{\mathrm{i}}, \mathrm{Q}_{\mathrm{co}}, \mathrm{P}_{\mathrm{z}}\right\}$ - размещение груза на судне, допуская неизменным значение $\mathrm{P}_{\mathrm{z}}$.

После выгрузки в первом порту оставшаяся загрузка судна обозначена $\mathrm{G}_{1}=\left\{\tilde{\mathrm{p}}_{2} \bigcup_{\mathrm{i} \neq 1} \mathrm{p}_{\mathrm{i}}, \mathrm{Q}_{\mathrm{c} 1}, \mathrm{P}_{\mathrm{z}}, \mathrm{P}_{\mathrm{b} 1}, \mathrm{Q}_{\mathrm{b} 1}\right\}$ или $\mathrm{G}_{1}=\left\{\mathrm{G}_{\mathrm{c} 1}, \mathrm{P}_{\mathrm{b} 1}, \mathrm{Q}_{\mathrm{b} 1}\right\}$. После выгрузки в предыдущих портах загрузка судна после сдачи груза в s-м порту формально выражается следующим образом:

$$
\mathrm{G}_{\mathrm{s}}=\left\{\mathrm{G}_{\mathrm{cs}}, \mathrm{P}_{\mathrm{bs}}, \mathrm{Q}_{\mathrm{bs}}\right\},
$$

причем $\mathrm{G}_{\mathrm{cs}}=\left\{\tilde{\mathrm{p}}_{\mathrm{s}+1} \bigcup_{\mathrm{i} \neq 1,2 . \mathrm{s}} \mathrm{p}_{\mathrm{i}}, \mathrm{Q}_{\mathrm{cs}}, \mathrm{P}_{\mathrm{z}}\right\}$.

Следовательно, каждая из загрузок судна $\mathrm{G}_{\mathrm{s}}$ перед выходом к очередному порту назначения должна обеспечить свободный доступ к грузу, предназначенному для выгрузки в следующем порту и удовлетворять требованиям мореходной безопасности $\mathrm{M}_{\mathrm{s}} \in \mathrm{Mn}_{\mathrm{sf}}$, как и допустимость сил инерции на качке $\mathrm{F}_{\mathrm{jks}}<\mathrm{F}_{\mathrm{jd}}$, на предстоящем переходе, что формально можно выразить следующим образом:

$$
\begin{aligned}
& \mathrm{G}_{\mathrm{s}}=\left\{\mathrm{G}_{\mathrm{cs}}, \mathrm{P}_{\mathrm{bs}}, \mathrm{Q}_{\mathrm{bs}}\right\}, \\
& \mathrm{G}_{\mathrm{cs}}=\left\{\tilde{\mathrm{p}}_{\mathrm{s}+1} \bigcup_{\mathrm{i} \neq 1,2 . . \mathrm{s}} \mathrm{p}_{\mathrm{i}}, \mathrm{Q}_{\mathrm{cs}}, \mathrm{P}_{\mathrm{z}}\right\}, \quad(\mathrm{s}=0 \ldots \mathrm{n}-1) \\
& \mathrm{M}_{\mathrm{s}} \in \mathrm{Mn}_{\mathrm{sf}}, \\
& \mathrm{F}_{\mathrm{jks}}<\mathrm{F}_{\mathrm{jd}},
\end{aligned}
$$

где $\mathrm{n}$ - число портов выгрузки. 
Для формирования последовательности загрузок судна $\mathrm{G}_{\mathrm{s}}$, удовлетворяющих перечисленным требованиям, следует учесть, что размещение груза $\mathrm{Q}_{\mathrm{cs}}$ в каждой загрузке судна включено в предыдущее $\mathrm{Q}_{\mathrm{c}(\mathrm{s}-1)}$. Следовательно, начальное размещение груза $\mathrm{Q}_{\mathrm{co}}$ содержит все последующие размещения $\mathrm{Q}_{\mathrm{cs}}$ и справедливы соотношения:

$$
\begin{aligned}
& \mathrm{G}_{\mathrm{cs}} \subset \mathrm{G}_{\mathrm{co}}, \quad(\mathrm{s}=0 \ldots \mathrm{n}-1) \\
& \bigcup_{\mathrm{s}=1}^{\mathrm{n}-1} \mathrm{G}_{\mathrm{cs}}=\mathrm{G}_{\mathrm{co}} .
\end{aligned}
$$

Поэтому формирование начальной загрузки $\mathrm{G}_{\mathrm{o}}$ необходимо начинать $\mathrm{c}$ определения допустимой последней загрузки $\mathrm{G}_{\mathrm{c}(\mathrm{n}-1)}$ перед сдачей груза в последнем порту, а затем пошагово перемещаться к предыдущей загрузке, пока не будет получена начальная загрузка в последнем порту загрузки.

При решении поставленной задачи следует учитывать связь между загрузкой судна и параметрами его качки, которые определяют характеристики возникающих угловых ускорений и влияют на величину сил инерции.

В работе [5] показано, что максимальное значение горизонтальной составляющей сил инерции $\operatorname{maxF}_{\mathrm{Hl}}$ при качке судна, действующей на контейнер определяется следующим выражением:

$$
\begin{gathered}
\operatorname{maxF}_{\mathrm{H} 1}=\mathrm{m}_{\mathrm{c}} \omega_{\mathrm{k}}^{2}\left\{\left[\Delta \mathrm{Z} \beta_{\mathrm{m}} \sin \left(\omega_{\mathrm{k}} \mathrm{t}-\psi_{\beta}\right)-\left(\frac{\mathrm{g}}{\omega_{\mathrm{k}}^{2}}+0,5 \mathrm{~h}_{\mathrm{w}} \sin \omega_{\mathrm{k}} \mathrm{t}\right) \cos \theta \sin \beta\right]^{2}+\right. \\
\left.+\left[\Delta \mathrm{Z} \theta_{\mathrm{m}} \sin \left(\omega_{\mathrm{k}} \mathrm{t}-\psi\right)+\left(\frac{\mathrm{g}}{\omega_{\mathrm{k}}{ }^{2}}+0,5 \mathrm{~h}_{\mathrm{w}} \sin \omega_{\mathrm{k}} \mathrm{t}\right) \sin \theta\right]^{2}\right\}^{1 / 2}
\end{gathered}
$$

где $\beta_{\mathrm{m}}$ - амплитудное значение угла дифферента $\beta$;

$\psi_{\beta}$ - начальная фаза продольных колебаний.

$\theta_{\mathrm{m}}$ - амплитудное значение угла крена;

$\psi$ - начальная фаза;

$\omega_{\mathrm{k}}$ - кажущаяся частота волнения;

$\mathrm{m}_{\mathrm{c}}$ - масса груза, $\Delta \mathrm{Z}$ - расстояние между центрами масс груза и судна по высоте.

Анализ приведенного выражения (1) показывает, что на значение $\max _{\mathrm{H} 1}$ набольшее влияние имеют размещение контейнера $(\Delta \mathrm{Z})$ и угловые ускорения бортовой качки, которые определяются величиной $\theta_{\mathrm{m}}$ :

$$
\theta_{\mathrm{m}}=\frac{\chi_{\theta} \omega_{\mathrm{o}}^{2}}{\left[\left(\omega_{\mathrm{o}}^{2}-\omega_{\mathrm{k}}^{2}\right)^{2}+4 \mathrm{~h}^{2} \omega_{\mathrm{k}}^{2}\right]^{1 / 2}},
$$

где $\omega_{\mathrm{k}}$ - кажущаяся частота волнения; 
$\omega_{\text {o }}$ - собственная частота судна при бортовой качке, причем

$$
\omega_{\mathrm{o}}^{2}=\frac{\mathrm{Dh}}{\left(\mathrm{J}_{\mathrm{x}}+\mathrm{m}_{\mathrm{x}}\right)},
$$

$\mathrm{h}_{\mathrm{o}}$ - поперечная начальная метацентрическая высота.

Минимизировать величину $\operatorname{maxF}_{\mathrm{H1}}$ до требуемых допустимых значений можно помимо размещения контейнера еще и уменьшением амплитудного значения угла крена $\theta_{\mathrm{m}}$, для чего, как следует из (2), необходимо добиться максимальной разницы между частотами $\omega_{\mathrm{o}}$ и $\omega_{\mathrm{k}}$. Если $\omega_{\mathrm{k}}$ является неуправляемой переменной и ее величину можно только прогнозировать на предстоящий переход, то величина $\omega_{\text {о }}$ зависит от загрузки судна, что следует из выражения (3). Именно загрузка судна определяет величину поперечной начальной метацентрической высоты $h_{\mathrm{o}}$ и момента инерции судна $\mathrm{J}_{\mathrm{x}}$ относительно продольной оси $\mathrm{X}-\mathrm{X}$. Если из множества допустимых загрузок выбрать ту, для которой $\left(\omega_{0}-\omega_{\mathrm{k}}\right)$ имеет максимальное значение, то при прочих равных условиях можно обеспечить выполнение требования допустимых значений сил инерции при качке судна.

\section{Выводы и перспектива дальнейшей работы по данному направлению}

Таким образом, рассмотрены основные особенности составления предварительного грузового плана контейнеровоза, вызванные спецификой его работы и влияющие на мореходную безопасность судна. В дальнейшем целесообразно исследовать зависимость сил инерции на качке от загрузки судна.

\section{ЛИТЕРАТУРА}

1. Чепок А.О. Оптимизация загрузки судна тарно-штучными грузами в автоматизированных системах / Чепок А.О. // Проблемы техники: Научнопроизводственный журнал / ОНМУ, ХНУ - 2013. - № 4. - Одесса: Одесский национальный морской университет, 2013. - С. 44-51.

2. Цымбал Н.Н. Формирование оптимизационной задачи проведения грузовых операций навалочных судов/ Цымбал Н.Н., Васьков Ю.Ю. // Судовождение. - 2004. - № 7. - С. 3 - 10.

3. Николаева Л.Л. Способ формализации формы судовых грузовых помещений нестандартного типа / Николаева Л.Л., Гайченя А.В. // Автоматизация судовых технических средств. - 2010. - № 16. - С. 71-82.

4. Власенко Е.А. Угловые ускорения, возникающие при качке судна/ Власенко Е.А.// Сучасні інформаційні та інноваційні технології на 
транспорті (MINTT-2018): Матеріали X Міжнародної наук.-практ. конф., 29-31 травня. 2018 - Херсон: ХДМА, 2018. - С. 96 - 99.

5. Власенко Е. А. Определение максимального значения горизонтальной составляющей сил инерций, действующей на груз при качке судна/ Власенко Е. А., Бурмака И. А.// Science and Education a New Dimension. Natural and Technical Sciences, VI (18), Issue: 158, 2018.- C. 80 - 84. 